\title{
A Theoretical and Experimental Analysis of Template Co-update in Biometric Verification Systems
}

\author{
Luca Didaci $^{1, *}$, Gian Luca Marcialis ${ }^{2, *}$, and Fabio Roli ${ }^{2, *}$ \\ * University of Cagliari, Italy \\ ${ }^{1}$ Department of Pedagogical and Philosophical Sciences \\ ${ }^{2}$ Department of Electrical and Electronic Engineering \\ Piazza d'Armi - I-09123 Cagliari (Italy) \\ \{luca.didaci, marcialis, roli\}@diee.unica.it
}

\begin{abstract}
Template update in biometric recognition system is aimed to improve the representativeness of available templates in order to make them adaptive to the large intra-class variations characterizing biometrics (e.g. fingerprints and faces). Among others, semi-supervised approaches to template update have been recently proposed. Since the lack of representativeness is due to the impossibility of sampling all possible variations of a given client biometric, these approaches exploit samples submitted during the recognition phase by adding the "highly genuine" ones to the related client gallery. In particular, the template co-update algorithm, which uses the mutual help of two complementary biometric matchers, has shown promising experimental results. However, no theoretical model has been proposed to explain the behaviour of the co-update algorithm and support the experimental results. This is the goal of this paper. Experimental results show the correctness of the proposed theoretical model.
\end{abstract}

\section{Introduction}

Biometric-based personal verification systems reached a good maturity degree, both scientifically and technologically. In particular, face and fingerprint are the most widely adopted biometrics [1-2].

However, advancements of the state of the art have been able only partially to solve crucial problems as the dependency of performance of the recognition algorithms from the behavioural and environmental conditions. In many cases, these variations are not easily predictable, because they can be related to short-time or medium-time causes. For example, a scratch on the finger skin or the face, or an unknown expression, or the so-called "aging" effect, which is crucial in performance of face recognition systems [1]. Although many efforts have been made to deal effectively with such problems, a well-founded and definitive solution has not yet been achieved.

With regard to recent works, the state of the art moved the above problems from the input patterns, which are difficulty predictable, to the patterns already stored into the system, which concur to define the client's "gallery": the so-called template(s). The template(s) is a sample, or a set of samples, which is considered "representative" 
of the subject appearance. In order to capture such templates, the "enrolment" (or "registration") session is performed, under the supervision of a human expert, with the goal of selecting the most appropriate templates according to pre-defined qualitative parameters (e.g. neutral expression, light equally distributed along the face, fingerprint ridge and valleys visually clear, etc.). Thus, the problem of facing with short-time and medium-time intra-class variations can be formulated as the problem of collecting representative templates of these variations [1]. However, it is widely acknowledged that capturing all possible subject variations during an enrolment session is impossible. On the other hand, the system performance strongly depends on such representativeness.

The first solution proposed to store representative templates has been proposed in [3] in a completely supervised manner. A novel and interesting approach to template selection and update can be referred as "semi-supervised" [4-9]. In this approach, samples collected during the system operations, thus "labelled" neither genuine users nor impostors, are exploited. In particular, samples showing very strong similarity to existing templates are added into the gallery of the related subject. The small variations introduced by these samples can gradually capture larger variations from other ones, so "attracting" samples which should be considered "difficult" when using only existing templates. This iterative process can increase the system robustness, leading to galleries truly representative of the subject appearance [4-9].

In particular, the authors proposed the "co-update" algorithm, which showed that the mutual "help" of two weakly correlated biometrics (e.g., fingerprints and faces) can increase the gallery representativeness more quickly than systems adopting only one biometric [7-8], thus improving the overall performance. Despite of these promising results, the co-update algorithm still lacks of a theoretical framework able to explain the behaviour of the algorithm, and, in particular, which are the "hidden" parameters that rule such behaviour. To this aim, we investigate the co-update algorithm by proposing a statistical framework for modelling the gallery size increase. The proposed model starts from hypotheses which fit the practical scenarios involving fingerprint and face biometrics. The aim is to give a first analytical framework able to point out the relationship between the amount of unlabelled data collected and the error rate reduction required for a certain application.

Experimental results on a chimerical data set show the reliability of the framework by comparing the gallery sizes predicted by the model with the gallery sizes obtained on test data.

The paper is organized as follows. Section 2 describes the proposed model. Section 3 reports the experimental results which validate the model. Section 4 concludes the paper.

\section{A Theoretical Framework for Template Co-update}

\subsection{The Template Co-update Algorithm}

The key idea behind the biometric co-updating is the exploitation of the mutual complementary performance of multi-modal biometrics [7]. In fact, the "complementary" performances of biometric matchers using distinct biometric traits, for example, face 
and fingerprint, are one of the fundamental motivations for multi-modal biometrics [10]. Intuitively, each recognizer is expected to assign correct labels to certain input data which are difficult for the other. In the case of co-update, two matchers are used (bi-modal system).

Let us suppose that we have a bi-modal biometric system made up of a face and a fingerprint recognition system, and a small set $D_{l}$ of biometric data, labelled with the users' identities, acquired during the enrolment session.

The set $D_{l}$ is used to create the initial template galleries. During the on-line operation of the biometric system, a batch $D_{u}$ (usually, much larger than $D_{l}$ ) of data is acquired. Each element of $D_{u}$ is a pair of biometric samples, i.e. face and fingerprint, from the same individual, so each biometric sample of the couple is characterized by the same true identity and by the same claimed identity (it is worth noting that we are not considering the possibility of fraudulent accesses to the system with fake biometrics, that is an open issue left for future works).

During the off-line co-update phase, each matcher is applied to the batch $D_{u}$ to verify the identity of each couple of biometric samples. A biometric sample is added to the set $D_{l}$ if and only if the identity of the other biometric sample of the couple has been verified. In other words, a face sample is added to the set $D_{l}$ if and only if the correspondent fingerprint sample's identity has been verified, and vice-versa.

In the enrolment (supervised) session, collect a set $D_{l}$ of face and fingerprint images.

A couple of face and fingerprint images is acquired for each user.

Create the fingerprint and face templates using the set $D_{l}$

Loop for $H$ iterations:

Collect a set $D_{u}$ without supervision. Each element of $D_{u}$ is a pair of face and

fingerprint samples $\left\{\mathbf{x}_{1}, \mathbf{x}_{2}\right\}$ from the same individual

For each biometry $i, i \in\{0,1\}$

Let $i$ be the 'master' biometry and $|1-i|$ be the 'slave' biometry

For each couple of elements in $D_{u}$

If the matcher 'master' verifies the claimed identity, the sample of the 'slave' biometry is added to the set $D_{l}$

Update face and fingerprint templates using the augmented labelled set $D_{l}$

Fig. 1. Co-updating algorithm. The main steps of the algorithm are shown.

It is worth noting that in the co-update process the two biometrics play complementary role. One biometry verifies the claimed identity, assuming the role of supervisor, and allowing the gallery of the other biometry to be augmented. Let us call "master" the biometry that assumes the supervisor's role, and "slave" the biometry whose gallery is augmented thanks to the master biometry. During the co-training process both biometrics assume, alternatively, master and slave roles. Both matchers are re-trained with this augmented data set (e.g., by updating the face and fingerprint template galleries), and the process is repeated a specified number of times.

Intuitively, co-updating is expected to work because each matcher may assign correct labels to certain examples, considered as difficult for the other one. Therefore, 
each matcher can increase the galleries size with examples which are very informative for the complementary one.

Insertion of new samples without external supervision might introduce incorrectly labelled samples in gallery, which becomes noise and will degrade the performance of the system. For this reason we need to choose "confident examples" using a very high co-update threshold value at which no false matches occur, namely, FAR $=0 \%$. Figure 1 shows the main steps of the algorithm.

Some observations can be made about this version of the algorithm. First of all, it is possible to use a lower threshold, at the cost of introduce into the galleries a certain number of 'impostors' [8]. This is a common problem in all co-update and self-update algorithms [8]. Moreover, the genuine example added to the gallery could be characterized by a low quality, providing no informative contribution to the gallery. This issue will be addressed in future work. The update method used in this work consists into add a new template to the gallery, but more sophisticated methods are possible, i.e. to obtain a new template "fusing" different examples [14].

\subsection{The Theoretical Framework}

Let $n$ be the number of different templates contained in a gallery, for a given identity. Let $N_{T O T}$ be the maximum number of templates that a gallery can contain. The role, master or slave, of the two biometrics, is highlighted by superscripts $\mathrm{M}$ and $\mathrm{S}$.

Some assumptions are made in order to propose a mathematically tractable model. The first assumption regards the finite discrete space used to represent different biometric impressions. Each identity can produce a maximum of $N_{T O T}^{(\text {face })}$ and $N_{T O T}^{(\text {fingerprint) }}$ different observations of the face and fingerprint biometrics, respectively. This assumption can be motivated easily in strongly controlled environments, where a small amount of substantial intra-class variations is admitted, but not collectable by the supervised enrolling. The main consequence of this assumption is that a new example acquired by the system does not necessarily lead to a substantial performance improvement, because the new example can be considered already present in the gallery. In the literature, it has been suggested to "fuse" such similar variations in order to obtain a super-template [6,9].

The second assumption states that all biometric impressions are equally probable (as usually stated in biometric applications), and the two traits are conditionally independent given the identity. This is motivated by the fact that no clear correlation has been found between the face appearance and the related client's fingerprint image.

In the co-update stage, a set $D_{u}$ of $\left|D_{u}\right|=k$ pairs of biometric samples (i.e. face and fingerprint) are presented to the system. As described in $\$ 2.1$, the 'master' biometry validates the claimed identity of correspondent samples of the 'slave' identity. Let us call $k$ ' the number of samples of the master biometry whose identity has been verified (i.e. is over the updating threshold in practice). Thus, $k$ ' is the number of samples of the slave identity that can be added to the slave gallery.

The value $k$ ' can be easily computed by considering that each of $k$ biometric samples of the master biometry can be considered as drawn with replacement from a homogeneous population of $N_{T O T}^{(M)}$ elements. The probability that a sample will be 
verified is $p=1-F R R^{(M)}$, where $F R R^{(M)}$ is the False Rejection Rate for the master biometry. The problem can be modeled using a binomial distribution, so the expected value of verified samples is E[\# verified samples $]=k p=k\left(1-F R R^{(M)}\right)$

Let us assume that for each co-update step the number of verified samples $k$ ' is equal to its expected value:

$$
k^{\prime}=k\left(1-F R R^{(M)}\right)
$$

It is worth noting that the effectiveness of the master biometry as supervisor towards the 'slave' biometry is related to its FRR value. If FRR=0 $(k$ ' $=k)$ the master biometry is equivalent to a "true" supervisor because it will verify the claimed identity of all 'genuine' samples in $D_{u}$. If FRR $>0$, then only $k$ ' $<k$ samples can be verified, thus only $k^{\prime}<k$ samples will be added to the slave gallery.

Since the two biometrics (face and fingerprint) are conditionally independent, from the point of view of the 'slave' biometry each of these $k$ ' elements can be considered as drawn with replacement from a homogeneous population of $N_{T O T}^{(S)}$ elements.

In order to calculate how many samples provide informative contribution to the gallery we are interested in the expected value of elements that are different among themselves and are not present in gallery.

Let us call $d$ the random variable representing the number of elements in $k$ ' that are different among themselves, and $d n$ the random variable representing the number of elements in $k$ ' that are different and not present in gallery.

The pdf $p_{d}(d)$ can be modeled using a multinomial distribution:

$$
p_{d}(d)=\left(\begin{array}{c}
N_{T O T} \\
d
\end{array}\right) \sum_{i=0}^{d}(-1)^{i}\left(\begin{array}{c}
d \\
i
\end{array}\right)\left(\frac{d-i}{N_{T O T}}\right)^{k^{\prime}} .
$$

The conditional pdf $p_{d n / d}(d n / d)$ - the probability to have $d n$ different samples not present in gallery if $d$ samples are different among themselves - can be modeled using an hyper-geometric distribution:

$$
p_{d n \mid d}(d n \mid d)=h\left(d n ; N_{T O T},\left(N_{T O T}-n\right), d\right) .
$$

where $N_{\text {TOT }}$ is the number of items in the population, $\left(N_{\text {TOT }}-n\right)$ the number of items in the population that are classified as 'highly confident genuine users', $d$ the number of items in the sample, $d n$ the number of items in the sample that are classified as 'highly confident genuine users'. Its expected value is $E[d n]=d\left(N_{\text {TOT }}-n\right) / N_{\text {TOT }}$.

Recalling (2) and (3), the pdf $p_{d n}(d n)$ can be written as:

$$
\begin{aligned}
p_{d n}(d n) & =\sum_{d=1}^{k^{\prime}} p_{d n / d}(d n / d) p_{d}(d)= \\
= & \left\{\begin{array}{cc}
\sum_{d=1}^{k^{\prime}}\left[\left(\begin{array}{c}
N_{T O T}-n \\
d n
\end{array}\right)\left(\begin{array}{c}
n \\
d-d n
\end{array}\right)\right]\left[\sum_{i=0}^{d}(-1)^{i}\left(\begin{array}{c}
d \\
i
\end{array}\right)\left(\frac{d-i}{N_{\text {TOT }}}\right)^{k^{\prime}}\right] & \text { if } d n \leq d \\
0 & \text { if } d n>d
\end{array}\right.
\end{aligned}
$$


The expected value $E[d n]$ represents the expected enlargement of the gallery due to the collection of $k$ ' elements. Let us assume that for each co-update step the number $\Delta n^{(S)}$ of new and distinct samples added to the slave gallery is about equal to its expected value $E[d n]$, since the correspondent variance $\operatorname{var}[d n]$ is very small. Recalling eq. (1):

$$
\Delta n^{(S)} \approx E[d n]=\sum_{d n=0}^{k^{\prime}}\left\{d n \cdot p_{d n}(d n)\right\}=\left(1-\left(1-\frac{1}{N_{T O T}^{(S)}}\right)^{k\left(1-F R R^{(M)}\right)}\right)\left(N_{T O T}^{(S)}-n^{(S)}\right) .
$$

Let $n_{0}^{(S)}$ be the initial size of the gallery, and $n_{(i)}^{(S)}$ the size of the gallery at the $i$-th co-update step. The length of the gallery at the $i$-th step can be written as:

$$
\begin{aligned}
& n_{(0)}^{(S)}=n_{0}^{(S)} \\
& n_{(i)}^{(S)}=n_{(i-1)}^{(S)} w+N_{T O T}^{(S)}(1-w)
\end{aligned} ; \quad w=\left(1-1 / N_{T O T}^{(S)}\right)^{k\left(1-F R R^{(M)}\right)} .
$$

The weight $w$ takes into account the effectiveness of the master gallery as a supervisor.

The FRR ${ }^{(\mathrm{M})}$ value determine the system's dynamics, so it is worth to relate its value to the length of the master gallery. In order to model FRR, let $m_{i}$ be the average number of examples that produce a 'score' over the updating threshold, if compared with a generic sample of the $i$-th client. Therefore, "difficult" clients can be modeled by a low value of $m_{i}$, whilst high value of $m_{i}$ is adopted for "easy" clients. With regard to this value, we can make the following observations. Let $N_{T O T}^{(M)}$ be the size of the user population for the master biometric:

1. If $m_{i}>\left(N_{T O T}^{(M)}-n^{(M)}\right)$, at least one of these $m_{i}$ examples is in the 'master' gallery, therefore, the claimed identity of the example $x$ will be verified (that is, their matching is above the updating threshold), and $\mathrm{FRR}^{(\mathrm{M})}=0$.

2. If $m_{i} \leq\left(N_{T O T}^{(M)}-n^{(M)}\right)$, the probability that exactly $r$ examples among the $m_{i}$ are in the gallery can be modeled using an hyper-geometric distribution $p(r)=h\left(r ; N_{T O T}^{(M)}, m_{i}, n^{(M)}\right)$. The probability $p(r=0)$ that no examples among the $m_{i}$ are in the gallery corresponds to the FRR value of the system:

$$
F R R^{(M)}=\left\{\begin{array}{ll}
0 & \text { if } m_{i}>\left(N_{T O T}^{(M)}-n^{(M)}\right) \\
h\left(0 ; N_{T O T}^{(M)}, m_{i}, n^{(M)}\right) & \text { if } m_{i} \leq\left(N_{T O T}^{(M)}-n^{(M)}\right)
\end{array} .\right.
$$

To sum up, eq. (6) allows us to model the gallery size increase during the coupdate iterations, whilst eq. (7) models the FRR of the master biometrics. These relationships can be used to predict the behaviour of co-update. In the next Section the experimental validation of the proposed model by eqs. (6-7) is done by co-updating a face and a fingerprint matcher on real data. 


\section{Experimental Results}

\subsection{The Data Set}

The data set adopted consists of 42 individuals composed of 20 face and fingerprint images for each individual, by keeping in mind the independence of face and fingerprint traits. The time span of both the collected data sets spans over one year. Fortytwo frontal face images with 20 instances representing significant illuminations changes and variations in facial expressions per person were used from the Equinox corporation database [13]. The fingerprint data set has been collected by the authors using Biometrika Fx2000 optical sensor. The images are acquired with variations in pressure, moisture and time interval to represent large intra-class variations. The results are computed on five random coupling of face and fingerprint datasets and are averaged. Whilst minutiae are simply extracted from the fingerprint images after commonly used processing algorithms [2], PCA is computed on the whole data set and applied to face images in order to reduce the size of the overall feature space. $95 \%$ of energy is retained according to the current literature [1].

It is worth noting that fingerprint and face data sets are strongly different in terms of environmental conditions: the face one is notably "simpler" than the fingerprint one. We adopted so different data sets in order to show the effect of intra-class variations on the model prediction ability.

\subsection{Experimental Protocol}

In order to investigate the correctness of the proposed model, we first implemented a simple bi-modal identification system made up of a PCA-based face matcher and a fingerprint matcher using the "String" matching algorithm ("String" is based on minutiae points). We used the standard versions of these two recognition algorithms [1112]. Then, we implemented the template co-update algorithm in Figure 1. Both the eigenspace of the PCA-based face matcher and the co-update threshold value at FAR $=0 \%$ are computed using the whole dataset, and has not been updated during the coupdate process. This approach is common to some template update practices [4-5]. The update of face and fingerprint templates is performed simply by adding new examples to the user's gallery.

In the experiments and in the model $m_{i}$ has been set as follows. We considered all samples for each client $i$, and computed $m_{i}$ as the average number of samples exceeding the updating threshold. Then, we selected, as the initial template in $D_{l}$, the image "near" to $m_{i}$ samples. The rationale for this choice is to exclude outliers from the initial gallery, likewise to what happens in real situations, where the initial template is chosen in completely supervised fashion.

In order to simulate the acquisition of a batch set $D_{u}$, several sets of $k=10$ couples face and fingerprint - of 'genuine' examples are drawn with replacement from a homogeneous population of $N_{T O T}^{(\text {face })}=N_{\text {TOT }}^{\text {(fingerprint })}=20$ samples, and used as batches collected during the system operations. We are aware that adopted database size may not be very well appropriate for the task, but it respects, on average, the size adopted in other template update works reported in literature [4-9]. 


\subsection{Experimental Results}

Figures 2-3 compare the gallery sizes predicted by the model (thin lines) with the gallery sizes obtained on test data (bold lines), for fingerprint and face biometrics, respectively. For each iteration of the algorithm depicted in Fig. 1, the size of the galleries is reported, whilst theoretical lines follow eqs. (6-7). Marked lines report gallery sizes averaged over all the identity. Largest and smallest gallery cases, related to clients exhibiting the largest and smallest $m_{i}$, are shown using dashed and solid lines.

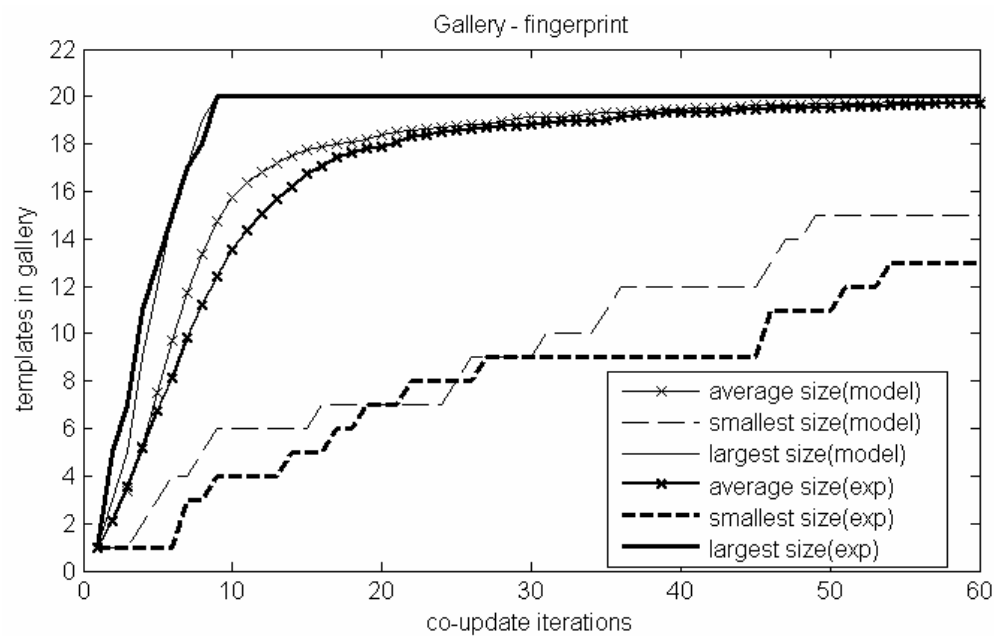

Fig. 2. Fingerprint galleries. Marked lines represent the gallery size averaged across all the identities. Dashed and solid lines represent the smallest and biggest galleries. Experimental trend (bold) and predicted values (thin) are shown.

The following observations can be drawn from Figures 2-3:

- the model is able to predict the experimental performance, with a negligible difference;

- prediction on the face matcher is the less accurate. This is due for sure to the strong intra-class variations which affect the fingerprint images. In other words, $m_{i}$ exhibits a variance so high that, on average, prediction becomes unstable especially at the initial iterations of the co-update algorithm. On the other hand, it can be noticed that, when focusing on single clients, the prediction is good even for the face matcher (thin and bold dotted lines);

- prediction on the fingerprint matcher is very good, even when evaluating the average behaviour of co-update. This is thanks to the good estimation of FRR of related face matcher, which takes the role of master-supervisor during the iterations. In other words, $m_{i}$ of clients faces exhibit a variance low enough. This is due to the fact that the face data set is "simpler" than the fingerprint one; 


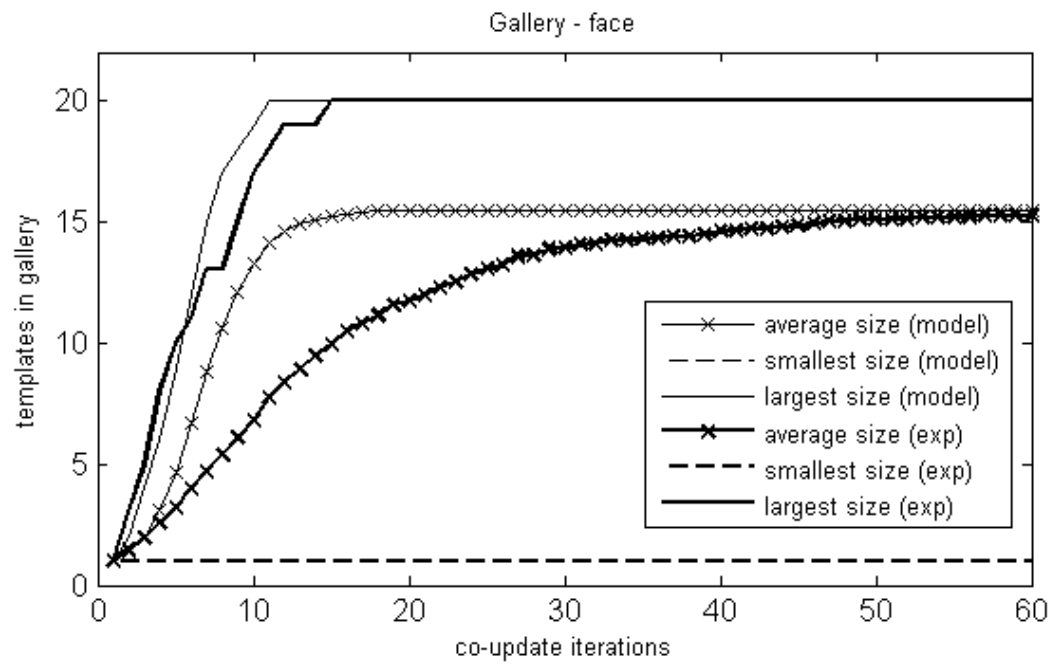

Fig. 3. Face galleries. Marked lines represent the galleries length averaged across all the identities. Dashed and solid lines represent the smallest and biggest galleries. Experimental trend (bold) and predicted values (thin) are shown.

- the saturation of theoretical and experimental curves is obviously due to the form of eqs.(6-7). In particular, the weight $w$ tends to decrease as the gallery size of both matchers increase, depending on the value of their FRR, thus the size of galleries must converge to $N_{T O T}$ if $m_{i}$ is not zero.

It is worth to point out that for 10 clients (details are omitted for brevity) the 'face' gallery cannot be enlarged by using the co-update algorithm, both in the model and in the experiments, because no fingerprint examples produce a 'score' over the updating threshold $\left(m_{i}=0\right)$. In this case, the reason lies in the high value of the global updating threshold that has been chosen. A user-specific threshold will permit to avoid this problem.

These good results must take into account some issues which have not yet been investigated here: (1) in real environments $N_{T O T}$ is unknown, accordingly an approach to estimate it is necessary. This problem can be overcame by considering $N_{T O T}$ as the number of samples in the batch exhibiting, for each client, a score above the zeroFAR threshold adopted. Worth noting, this assumption does not consider the problem of impostors insertion, a still open issue as remarked in [9]; (2) the same for the estimation of $m_{i}$. Therefore, in a future work, we will investigate the impact of $N_{T O T}$ and $m_{i}$ predicted on a batch of samples including even impostors, on a larger, non chimerical data set.

\section{Conclusions}

In this paper, we proposed a theoretical framework able to model the behaviour of the co-update algorithm. The co-update method appears very promising for galleries and 
performance improvement, because it exploits the complementary characteristics of two matchers.

The framework works on the basis of some assumptions which are, in our opinion, reasonable for biometrics in real applications. The reported experimental validation of the model showed that its prediction ability is good even in the case of a strong performance difference among matchers, due to intra-class variations in the data.

Although we pointed out some issues which will be clarified or solved in future works, we believe that this model can be considered as a good starting point for the theoretical investigation of the co-update algorithm behaviour.

\section{References}

1. Li, S.Z., Jain, A.K. (eds.): Handbook of face recognition. Springer, Heidelberg (2005)

2. Maltoni, D., Maio, D., Jain, A.K., Prabhakar, S.: Handbook of fingerprint recognition. Springer, Heidelberg (2003)

3. Uludag, U., Ross, A., Jain, A.K.: Biometric template selection and update: a case study in fingerprints. Pattern Recognition 37(7), 1533-1542 (2004)

4. Jiang, X., Ser, W.: Online Fingerprint Template Improvement. IEEE Trans. PAMI 24(8), 1121-1126 (2002)

5. Ryu, C., Hakil, K., Jain, A.: Template adaptation based fingerprint verification. In: Proc. of ICPR, vol. 4, pp. 582-585 (2006)

6. Roli, F., Marcialis, G.L.: Semi-supervised PCA-based face recognition using self-training. In: Yeung, D.-Y., Kwok, J.T., Fred, A., Roli, F., de Ridder, D. (eds.) SSPR 2006 and SPR 2006. LNCS, vol. 4109, pp. 560-568. Springer, Heidelberg (2006)

7. Roli, F., Didaci, L., Marcialis, G.L.: Template co-update in multimodal biometric systems. In: Lee, S.-W., Li, S.Z. (eds.) ICB 2007. LNCS, vol. 4642, pp. 1194-1202. Springer, Heidelberg (2007)

8. Roli, F., Didaci, L., Marcialis, G.L.: Adaptive biometric systems that can improve with use. In: Ratha, N., Govindaraju, V. (eds.) Advances in Biometrics: Sensors, Systems and Algorithms, pp. 447-471. Springer, Heidelberg (2008)

9. Rattani, A., Marcialis, G.L., Roli, F.: Capturing large intra-class variations of biometric data by template co-updating. In: IEEE Workshop on Biometrics, Int. Conf. on Vision and Pattern Recognition CVPR 2008, Anchorage, Alaska, USA (2008) (in press)

10. Ross, A., Nandakumar, K., Jain, A.K.: Handbook of Multibiometrics. Springer, Heidelberg (2006)

11. Jain, A.K., Hong, L., Bolle, R.: On-line Fingerprint Verification. IEEE Transactions on Pattern Analysis and Machine Intelligence 19(4), 302-314 (1997)

12. Turk, M., Pentland, A.: Eigenfaces for Face Recognition. Journal of Cognitive Neuroscience 3(1), 71-86 (1991)

13. http://www. equinoxsensors. com/products/HID.html

14. Freni, B., Marcialis, G.L., Roli, F.: Replacement algorithms for fingerprint template update. In: Campilho, A., Kamel, M.S. (eds.) ICIAR 2008. LNCS, vol. 5112. Springer, Heidelberg (in press, 2008) 